\title{
Development History and Prospect of TCM Psychology
}

\author{
Huajie Sui, Xunbing Shen* \\ School of Humanities, Jiangxi University of Traditional Chinese Medicine, Nanchang, 330001, China \\ *Corresponding author
}

Keywords: TCM psychology; development history; prospect; emerging discipline.

\begin{abstract}
Psychology of traditional Chinese medicine (TCM) is a new discipline which aims to study the relation between psychology and body based on TCM theory, and it owns Chinese characteristics. According to the development of TCM psychology, a systematic discipline system forms, ranging from its birth in 1985 to its development to "seven theories" from "six theories" and the final ideological transition of TCM psychology. In the end, the hard course of TCM psychology development is analyzed scientifically, and its devilment prospect is associated on the basis of rational imagination.
\end{abstract}

\section{Introduction}

The first TCM psychology seminar was convened smoothly in 1985, and the discipline of TCM psychology rose. This further improved medical system of China. The birth of TCM psychology made the quintessence of Chinese culture - TCM and modern medicine penetrate into each other and closely combine each other. Therefore, TCM psychology owns two attributes: theory and clinical practice. "Six theories" in TCM psychology refer to personality craseology, integration of body and spirit, psychology controlled by mind, perception by mind, emotions influenced by internal organs and relation between dream and sleep. From the "six theories" to "seven theories", this reflects the holistic view of life and environment, and illuminates basic views of TCM psychology. Based on "six theories", TCM personality theory with national characteristics (i.e. "Heaven, earth and human" holism) is evolved. At present, 11 academic conferences about TCM have been held in China, which makes TCM psychology form a relatively thorough system. With the help of the state, individual researches (such as "liver depression") have reached breakthroughs. The development of TCM is rapid, and it has risen since 1985. Now, its development trend cannot be resisted.

\section{Research purpose of TCM psychology}

In recent years, the colleagues of TCM, western medicine and psychology circles have done large quantities of work to sort out the materials of TCM psychology, and conducted lots of researches and explorations based on the analysis and statistical data. Their research purpose is mainly reflected in three aspects: to relieve human pains caused by diseases and improve treatment effect; to enhance health maintenance function and achieve the purpose of nourishing heart and living long; to explore profound meanings of TCM, give full play to the functions of TCM and perfectly combine TCM with modern psychology so as to benefit all human beings.

\section{Early history of TCM psychology}

At the beginning of 1950s, the experts of Beijing University of Chinese Medicine and Fujian TCM Hospital took the lead to sort out and conclude classical medical books and preliminarily integrated TCM with modern medicine. Then, a systematic TCM discipline formed. It was soon included in the class schedule of universities and colleges. In 1980, Wang Miqu - doctoral supervisor of Beijing University of Chinese Medicine and others proposed a new concept of TCM psychology. Then, 
through a series of researches, TCM psychology was born on the first national TCM psychology seminar in Chengdu in 1985. In the middle 1990s, the scholars achieved innumerable great achievements about the research on psychology so that the research on TCM psychology reached a false saturation condition. Seeing from their research achievements, Huangdi Neijing is the core, and the idea of union of mind and body is penetrated in it. Besides, it is consistently embodied in the whole theoretical framework. These theories gained by combining historical analysis are very creative. The unique thought of TCM psychology they express can form a system which can be scientifically applied in medicine to promote the progress of modern medicine.

Nowadays, although Dong Jianhua, Ma Peng and Wang Shenglong have passed away, groups of promising young people are rising sharply. If Dong Jianhua, Ma Peng and Wang Shenglong are the pioneers of this medicine path, the groups of comers who are becoming old form young are the leaders of this path. It is their existence that makes the development of TCM psychology free from various obstructions and continuous. To respond to the call of Ministry of Health, the researches on TCM psychology strive for further improvement under the support of State Administration of Traditional Chinese Medicine of the P.R.C, which can be seen from the great increase in relevant papers and monographs. Moreover, the development of TCM psychology is not just longitudinal but also horizontal, which can be seen from the rise of TCM colleges and universities like bamboo shoots in spring, and the support of scientific research on TCM psychology by the state.

The first work of TCM psychology was completed in 1985. The work TCM Psychology was written and published by Prof. Wang Miqu of Chengdu University of TCM, and the preface was written by Mr. Fang Yaozhong. The writing thought of the book presents the strong principle. It pays equal attention to classroom theory and clinical operation from the beginning to the end, and focuses on improvement of clinical guidance. Besides, it concentrates on improvement of clinical guidance value of TCM psychology. The disperse chapters are organized, and the pace is also grasped rationally. The breadth and depth of theoretical explanation are also achieved. Meanwhile, basic theoretical knowledge is closer related to clinical practice, which perfectly improves readability and practical value of the book.

\section{Later history of TCM psychology}

In June 2006, the $11^{\text {th }}$ "Founding Conference of TCM Psychology Committee of World Federation of Chinese Medicine Societies (WFCMS)-Academic Conference of International TCM Psychology" was convened. This conference is very special, because it not only attracts TCM experts from Mainland China, Taiwan, Hong Kong and Macao, but also collects overseas papers about TCM psychology (dominated by modern psychology and supplemented by TCM) such as US, Germany, Australia, UK and Russia. Meanwhile, the papers from Singapore, Malaysia, Thailand, Japan and Korea are also gathered. According to the statistics, there are nearly 300 papers. It can be said that, this is a great cultural feast in TCM academic circle. After every national TCM psychology seminar ends, a group of excellent scholars of TCM psychology will be organized to write Symposium of TCM Psychology, and it will be publicly published by Singapore medicine and health.

After the conference in June 2006 ended, the Collected Papers was named International Symposium of TCM Psychology rather than Symposium of TCM Psychology. It is a book of papers with the largest number of papers, the most disputes and the most extensive researches collected by TCM psychology seminar since the birth of Symposium of TCM Psychology. Although the quality of papers is uneven, it improves significantly. Generally speaking, this summarizes the bumpy way of TCM psychology over the past 21 years.

Wang Keqin (1941) - the honorary chairman of World Federation of Chinese Medicine Societies (WFCMS) and editorial board member of Yearbook of Chinese Traditional Medicine (academic volume) expanded and improved the framework of original "six theories" to form "seven theories" in 2008. Based on the research ideas of Yan Shangao, Zhu Wenfeng, Min Fanzhong, Zhang Xiaojuan, Huang Xiaoling and other scholars, Prof. Wang Keqin conduced in-depth researches and combined the discussion that living environment directly influences human physiological activities in Huangdi 
Neijing (i.e. refer to Su Wen · Si Qi Shen Diao Da Lun and Su Wen · Yi Fa Fang Yi Lun) to carefully expound the influence of four seasons and each direction. After further thinking, Prof. Wang Keqin studied the rules of sleep from the important TCM work Zhen Jing. In other words, through analyzing the changes of Wei Qi of Yin and Wei Qi of Yang, he found the daily routines of wild animals which work in the daytime and sleep in the nighttime are also suitable for human. Besides, wind, rain, weather, timbre and smell have effects on human psychological spirit. Through specializing in Neijing, Pro. Wang Keqin has thoroughly analyzed the profound meanings, and applied these profound meanings to construct the framework of "seven theories".

The proposal of "seven theories" can be regarded as the small progress in the later period of TCM psychology. Since the first national academic conference of TCM psychology was convened in Chengdu in 1985, "six theories" were first proposed and On Foundation of TCM Psychology was published, the prototype of "six theories" has appeared. After the basic theoretical framework was constructed, it was fully embodied in teaching material preparation, some monographs or papers. Most importantly, it also gained the sufficient affirmation of many experts. For example, the expert group led by the director Fang Yaozhong evaluated that, "six theories" not just follow the theoretical system of TMC, but also develop and innovate academically and lay a solid theoretical foundation for clinical application of TMC psychology. Hence, we can imagine that, "six theories" to TMC psychology is just like golden cudgel to Sun Wukong. Just because of "six theories", the construction and development of TCM psychology can proceed smoothly. However, with the appearance of "seven theories" 23 years later, although the fact has proven that the framework of "six theories" can stand the test of time, this does not influence the progress of "seven theories" and its influence at all.

\section{Development prospect of TCM psychology}

Although TCM psychology is an emerging discipline, TCM is extensive and profound, with a long history. The thought of TCM psychology is long-standing and can be traced back to the Eastern Han Dynasty, which can be seen from the records in Huangdi Neijing. According to the statistics of relevant departments, Neijing is the favorite in the eyes of TCM psychology experts. In combination of the discussion on psychology and medicine in Neijing, there are 32 articles about psychology and medical psychology (involving the title, theme and main content) in International Symposium of TCM Psychology, accounting for 1/9 of the whole book. Thus, the influence of Huangdi Neijing on TCM medicine is very great. In addition, Huangdi Neijing has gone through the test of time, so TCM psychology based on Huangdi Neijing will also stand the test of time.

In one word, the rise of TCM psychology is not occasional or lucky. It has the profound foundation, so it is an emerging discipline. It also can be deemed as an "old" discipline. With rich and ancient psychotherapy methods and excellent treatment effect, TCM psychology also provides flexible clinical means for treatment of psychosomatic diseases. Looking back the development history of TCM psychology, we can see that through the efforts of numerous hardworking scholars, the academic status of TCM psychology has existed in TCM and modern psychology. Although it has achieved many achievements such as the research on "fright impairing kidney" and "liver depression". Under the financial help of Natural Science Foundation of China, TCM psychology has gained breakthroughs in individual aspects. In the future, more dazzling sparks will be generated in the combination of TCM and modern psychology. Future experts of TCM and modern psychology will move ahead in the interdiscipline of TCM and psychology on the basis of Huangdi Neijing. They will integrate the essence of TCM in psychological process and individual mind of psychology. The psychological process includes cognition process, emotion process and will process. Individual mind is human nature tendency and personality ability. Looking back personality craseology, integration of body and spirit, psychology controlled by mind, perception by mind, emotions influenced by internal organs and relation between dream and sleep, we can know that the integration of TCM and psychology is a certain event sooner or later. 


\section{Conclusion}

In the era where science develops rapidly, the research and development of TCM psychology are crucial. To make people keep healthy and own good spiritual state while creating a good world of material, the research on TCM psychology is pressing. Seeing from the current research course, theoretical research of TCM psychology is encountered an important historical moment. To let beginners and researchers have a profound cognition of its research significance, it is very necessary to clearly analyze the past, present and future of theoretical research on TCM psychology. Only when we clearly understand TCM psychology can it sustainably develop and finally benefit people.

\section{Acknowledgement}

This project is funded by Art Planning Program of Culture Department of Jiangxi Province (No.: YG20163398).

\section{References}

[1] Wang Miqu, Fu Xingguo, Chen Guiting, Preliminary Exploration of TCM Psychology, Journal of Chengdu University of Traditional Chinese Medicine, 1980, (3):12.

[2] Wang Keqin, On Foundation of TCM Psychology, Symposium of TCM Psychology, 1986,2(2):8.

[3] Wang Gang, Hu Guoku, Wang Miqu et al., Study on Differential Expression Gene of Rates with Deficiency Kidney by Suppression of Subtractive Hybridization. Journal of Beijing University of Traditional Chinese Medicine, 2003, (2):45-47.

[4] Li Jia, Zhang Hui, Yu Xiaoping et al., Study on "Qi" Measurement and Five Theories of Qi Function in Neijing-Theoretical Foundation of Line Drawing Test of Qi Function, Journal of Liaoning University of Traditional Chinese Medicine, 2010, 12(5): 21-22. 